\title{
A theory for multiple partially massless spin-2 fields
}

\author{
Lucas Traina* \\ Service de Physique de l'Univers, Champs et Gravitation, Université de Mons, UMONS \\ Research Institute for Complex Systems, Place du Parc 20, 7000 Mons, Belgium \\ E-mail: lucas.traina@umons.ac.be \\ Nicolas Boulanger \\ Service de Physique de l'Univers, Champs et Gravitation, Université de Mons, UMONS \\ Research Institute for Complex Systems, Place du Parc 20, 7000 Mons, Belgium \\ E-mail: nicolas.boulanger@umons.ac.be
}

\section{Cédric Deffayet}

Sorbonne Université, UPMC Paris 6 and CNRS, UMR 7095, Institut d'Astrophysique de Paris, GReCO, 98bis Boulevard Arago, 75014 Paris, France

IHES, Le Bois-Marie, 35 Route de Chartres, 91440 Bures-sur-Yvette, France

E-mail: deffayet@iap.fr

\section{Sebastian Garcia-Saenz}

Theoretical Physics, Blackett Laboratory, Imperial College, London, SWr 2AZ, U.K.

E-mail: s.garcia-saenz@imperial.ac.uk

\begin{abstract}
In this note, we review the results of the letter arXiv:1906.03868 where we revisited the problem of constructing consistent interactions for a set of partially massless spin-2 fields in (anti-)de Sitter spacetime of arbitrary dimension. This was done using the BRST-BV deformation procedure around that background. As a result of the analysis, we recovered the impossibility of building a consistent theory of a single partially massless spin-2 field and were able to construct a completely non-linear theory for a multiplet of such fields that is consistent from the gauge invariance point of view, although having negative relative signs between kinetic terms.
\end{abstract}

Corfu Summer Institute 2019 "School and Workshops on Elementary Particle Physics and Gravity" (CORFU2019)

31 August - 25 September 2019

Corfù, Greece

${ }^{*}$ Speaker. 


\section{Introduction}

Partially massless (PM) fields of spin-2 have been subject to renewed attention in recent years after the important advances made in the understanding of massive gravity $[1,2]$. In four-dimensional de Sitter $\left(\mathrm{dS}_{4}\right)$ spacetime with (positive) cosmological constant $\Lambda$, a PM graviton has a mass given by $m_{\mathrm{PM}}^{2}=2 \Lambda / 3$ and a corresponding gauge invariance that removes the degree of freedom associated with the spin-zero mode of the particle. A consistent theory of PM gravity would be very attractive given its relevance in the context of cosmology and the fact that a PM spin-2 field is not subject to the same strict experimental constraints as a generic massive graviton, e.g. the bounds on fifth-force experiments or on dispersion of gravitational waves.

In spite of their potentially interesting phenomenology, complete and physically realistic models involving PM fields are currently lacking. A crucial hurdle one encounters when attempting to construct such a PM theory beyond linear level is the requirement of gauge invariance of the interactions. Indeed, this condition is restrictive enough to rule out theories of a single self-interacting PM spin-2 particle, see e.g. [3, 4, 5]. Such no-go results beg the question of whether a fundamental obstruction exists for the construction of non-trivial PM models. A first step in order to address this question is to precisely understand what are the assumptions that lead to the existing negative results. In order to do so, we use the cohomological reformulation [6] in the BV formalism [7, 8] of the deformation procedure [9]. Indeed, this method allows to classify all the possible interactions starting from a given theory which relies on clearly defined assumptions.

In the recent work [10] we have shown that, by relaxing the requirement of classical unitarity $^{1}$, one can in fact construct a complete theory for a multiplet of PM gravitons around $(\mathrm{A}) \mathrm{dS}_{4}$ space, as we review in the following. This is an interesting outcome as it demonstrates that gauge invariance itself is not a fundamental obstacle for the existence of a non-trivial interacting PM spin-2 theory.

\section{BRST-BV deformation procedure}

The cohomological reformulation of the deformation procedure exposed in [9] was proposed in [6]. It exploits the antifield formalism [7, 8] for gauge theories. A detailed and accessible introduction to the BRST-BV deformation procedure can be found in [12]; see also the introduction of [13]. Here we reproduce the essential aspects in order to fix the necessary notation and describe the main steps of our approach in a self-contained manner as possible.

Consider a theory for a set of gauge fields $\varphi^{i}$ defined by an action $S\left[\varphi^{i}\right]$ that is invariant under the infinitesimal gauge symmetries

$$
\delta_{\epsilon} \varphi^{i}=R_{\alpha}^{i} \epsilon^{\alpha},
$$

\footnotetext{
${ }^{1}$ Actually, PM fields can be defined around $D$-dimensional Anti-de Sitter $\left(\operatorname{AdS}_{D}\right)$ spacetime where they are classically non-unitary. This can be seen explicitly by writing the action, which is real for both signs of the cosmological constant, in the Stueckelberg formulation [11].
} 


\begin{tabular}{lccc}
\hline & gh & antifld & puregh \\
\hline \hline Fields $\varphi^{i}$ & 0 & 0 & 0 \\
Ghosts $C^{\alpha}$ & 1 & 0 & 1 \\
Antifields $\varphi_{i}^{*}$ & -1 & 1 & 0 \\
Ghost antifields $C_{\alpha}^{*}$ & -2 & 2 & 0 \\
\hline
\end{tabular}

Table 1: Ghost, antifield and pureghost quantum numbers.

where, using De Witt's notation, a summation over repeated indices also means that an integral over an omitted dummy coordinate is implied, so that (2.1) gives a local relation depending on the gauge parameters $\epsilon^{\alpha}$ and their derivatives up to some finite order. The operator $R_{\alpha}^{i}$ may depend on the gauge fields and some of their successive derivatives.

In the BRST formalism $[14,15]$, one proceeds by associating a ghost field $C^{\alpha}$ to every gauge parameter $\epsilon^{\alpha}$, with a shift in the Grassmann parity: $\left|C^{\alpha}\right|=\left|\epsilon^{\alpha}\right|+1(\bmod 2)$. If the theory is reducible, such as for $p$-form gauge theories with $p>1$, one also introduces a hierarchy of higher-degree ghosts (ghosts of ghosts). We will not discuss these cases here and stick to irreducible gauge theories. In the antifield extension of the BRST formalism due to Batalin and Vilkovisky, to the gauge fields $\varphi^{i}$ and the BRST ghosts $C^{\alpha}$ one associates the antifields $\varphi_{i}^{*}$ and $C_{\alpha}^{*}$, respectively. For concreteness we will take both the fields $\varphi^{i}$ and the gauge parameters $\epsilon^{\alpha}$ to be bosonic, thereby excluding the discussion of supergravity theories (see $[16,17]$ for examples in that case), although the general case can be treated in much the same way modulo some obvious changes (see for example the pedagogical review [18]). In this situation the ghost antifields are Grassmann-even or commuting variables as well, while the ghosts $C^{\alpha}$ and antifields $\varphi_{i}^{*}$ are Grassmann-odd or anticommuting. Next we introduce two gradings, the ghost number "gh" and the antifield number "antifld", according to table 2. It is also useful to keep track of the number of differentiated or undifferentiated ghosts (not counting the associated antifields), via the pureghost number "puregh". We collectively denote the fields and ghosts by the variables $\Phi^{A}$, while their associated antifields are denoted by $\Phi_{A}^{*}$.

To the initial theory with gauge-invariant action $S\left[\varphi^{i}\right]$, one associates a BV functional $W\left[\Phi^{A}, \Phi_{A}^{*}\right]$ in the following way:

$$
W\left[\Phi^{A}, \Phi_{A}^{*}\right]=S\left[\varphi^{i}\right]+\varphi_{i}^{*} R_{\alpha}^{i} C^{\alpha}+\frac{1}{2} C_{\gamma}^{*} f_{\alpha \beta}^{\gamma} C^{\alpha} C^{\beta}+\frac{1}{4} \varphi_{i}^{*} \varphi_{j}^{*} M_{\alpha \beta}^{i j} C^{\alpha} C^{\beta}+\cdots
$$

By construction, as it is required to start with the classical action and to have definite ghost number and Grassmann parity, the BV functional $W$ is defined to be bosonic (Grassmanneven) with ghost number zero. More importantly, it is required to satisfy what is called the classical master equation:

$$
(W, W)=0
$$


where the antibracket, also called BV bracket, is defined by ${ }^{2}$

$$
(A, B)=\frac{\delta^{R} A}{\delta \Phi^{A}} \frac{\delta^{L} B}{\delta \Phi_{A}^{*}}-\frac{\delta^{R} A}{\delta \Phi_{A}^{*}} \frac{\delta^{L} B}{\delta \Phi^{A}} .
$$

At zero antifield number, the master equation yields the Noether identities associated with the original gauge symmetries of the action,

$$
\frac{\delta S}{\delta \varphi^{i}} R_{\alpha}^{i}=0
$$

Next, the terms with antifield number one in (2.3) produce

$$
R_{\alpha}^{j} \frac{\delta R_{\beta}^{i}}{\delta \varphi^{j}}-R_{\beta}^{j} \frac{\delta R_{\alpha}^{i}}{\delta \varphi^{j}}=R_{\gamma}^{i} f_{\alpha \beta}^{\gamma}+\frac{\delta S}{\delta \varphi^{j}} M_{\alpha \beta}^{j i}
$$

which is nothing but the gauge algebra of the transformations (2.1), which gives the interpretation of $f_{\alpha \beta}^{\gamma}$ as "structure functionals", since in general they are operators that may depend on the fields. The presence of the term proportional to $M_{\alpha \beta}^{i j}$ implies that the algebra will in general be "open", meaning that two gauge transformations will only close upon use of the equations of motion. Similarly, at the following order one finds the Jacobi identity satisfied by the structure functionals, and continuing in this manner generates a tower of consistency relations involving the higher order tensors - the ellipsis in (2.2) that characterize the gauge group of the theory. We refer to [18] for detailed discussions and review.

The main idea of the deformation analysis is to revert this story. The full action $S$ and its gauge symmetries are unknown, and we seek to determine them by perturbatively solving the master equation (2.3), knowing an initial action $S_{0}$ invariant under the gauge transformations

$$
\delta_{0} \varphi^{i}=R_{0}{ }^{i}{ }_{\alpha} \epsilon^{\alpha} .
$$

The BV functional, as we recalled, encodes all the information about the gauge structure of the theory. Hence the BV formalism is equivalent to other more direct approaches that aim at determining a theory based on the action and gauge transformation, although we will see that it is in many ways more powerful.

In order to solve the classical master equation, we consider the functional $W$ as a perturbation series in some overall deformation parameter $g$, i.e.,

$$
W=W_{0}+g W_{1}+g^{2} W_{2}+\cdots .
$$

Here $W_{0}$ corresponds to the BV functional associated with the theory that is already known and that one wishes to deform perturbatively. In the scenario we will focus on, the

${ }^{2}$ Right and left functional derivatives are defined via

$$
\delta A=\int \frac{\delta^{R} A}{\delta \Phi^{A}} \delta \Phi^{A}+\int \frac{\delta^{R} A}{\delta \Phi_{A}^{*}} \delta \Phi_{A}^{*}=\int \delta \Phi^{A} \frac{\delta^{L} A}{\delta \Phi^{A}}+\int \delta \Phi_{A}^{*} \frac{\delta^{L} A}{\delta \Phi_{A}^{*}} .
$$

The distinction between left and right derivatives of course only makes a difference when the derivative is with respect to a fermionic (Grassmann-odd) variable. 
known theory will be free, so that the deformation procedure amounts to the study of the consistent interaction vertices one can add to it. However, the formalism is not restricted to this case, since for instance one can also apply it to known models which are themselves already interacting, see for example $[19,20]$ for recent analyses.

The master equation approach becomes particularly powerful when rephrased as a cohomological problem [6]. The BV functional $W_{0}$ for the free theory $S_{0}$ is viewed as the generator of BRST transformations, in the sense that

$$
s A:=\left(W_{0}, A\right),
$$

for any local functional $A$, with $s$ denoting the BRST differential of the free theory. By a local functional $A$, one means the integral $\int a$ of a $D$-form $a$ that depends on the fields (including the ghosts), their associated antifields and their derivatives up to some arbitrary but finite order, which we indicate by the notation $a=a\left([\Phi],\left[\Phi^{*}\right], d x\right)$. Moreover, in this work we assume that all the fields and their derivative vanish at infinity, or alternatively that they have compact support, which enables us to discard all boundary terms. With this assumption, any local $D$-form $a\left([\Phi],\left[\Phi^{*}\right], d x\right)$ is equivalent to $a\left([\Phi],\left[\Phi^{*}\right], d x\right)+d b\left([\Phi],\left[\Phi^{*}\right], d x\right)$ where $b\left([\Phi],\left[\Phi^{*}\right], d x\right)$ is a local $(D-1)$-form and where $d$ is the total exterior differential $d=d x^{\mu} \partial_{\mu}$, where $\partial_{\mu}=\frac{\partial}{\partial x^{\mu}}+\partial_{\mu} z^{M} \frac{\partial}{\partial z^{M}}+\ldots$ is the total derivative that takes into account the spacetime dependence of the fields and antifields that we have collectively denoted by $z^{M}:=\left(\Phi^{A}, \Phi_{A}^{*}\right)$. We use conventions whereby $s$ anticommutes with $d$, or equivalently, for the type of theories we will deal with, $d x^{\mu} C^{\alpha}+C^{\alpha} d x^{\mu}=0$ and $d x^{\mu} \varphi_{i}^{*}+\varphi_{i}^{*} d x^{\mu}=0$. One has the following isomorphism of cohomological classes, $H^{g}(s) \cong H^{g, D}(s \mid d)$, where $H^{g}(s)$ denotes the cohomology of $s$ in the class of ghost number $g$ local functionals and $H^{g, D}(s \mid d)$ is the cohomology of the differential $s$, at ghost number $g$, in the space of local $D$-forms. In other words, the cohomology class in $H^{g, D}(s \mid d)$ is defined up to the $\sim$ equivalence relation by a representative solution $a^{g, D}$ of

$$
s a^{g, D}+d a^{g+1, D-1}=0, \quad a^{g, D} \sim a^{g, D}+s b^{g-1, D}+d b^{g, D-1} .
$$

The first superscript refers to the ghost number and the second one to the form degree.

The basic properties of the BRST differential $s$ then follow from the properties of the antibracket; in particular the nilpotency, $s^{2}=0$, is a consequence of the (graded) Jacobi identity and of the master equation $\left(W_{0}, W_{0}\right)=0$.

Given a free theory with action $S_{0}\left[\varphi^{i}\right]$ invariant under irreducible gauge symmetries as in (2.7), we can write the effect of a BRST transformation on the fields by decomposing $s=\gamma+\delta$, such that

$$
\gamma \varphi^{i}=R_{0}{ }^{i}{ }_{\alpha} C^{\alpha}, \quad \delta \varphi_{i}^{*}=\frac{\delta S_{0}}{\delta \varphi^{i}}, \quad \delta C_{\alpha}^{*}=\varphi_{i}^{*} R_{0}{ }^{i}{ }_{\alpha},
$$

while the action of $\gamma$ and $\delta$ on the variable not shown is by definition zero. It follows immediately that

$$
\gamma^{2}=0, \quad \delta^{2}=0, \quad \gamma \delta+\delta \gamma=0,
$$

which together again amount to the nilpotency of $s$. We recall that, with our conventions, $\{\gamma, d\}=0=\{\delta, d\}$. Observe that $\delta$ decreases the antifield number by one unit, while $\gamma$ does 
not change it. From eqs. (2.3) and (2.8), one has that the master equation, up to order $g^{2}$, yields

$$
\begin{aligned}
& s W_{0}=0, \\
& s W_{1}=0, \\
& s W_{2}=-\frac{1}{2}\left(W_{1}, W_{1}\right) .
\end{aligned}
$$

The first of this holds trivially of course since the free action $S_{0}$ and its gauge invariance is known. The second equation then states that the first order deformation of the BV functional is BRST-closed. Any BRST-exact expression $W_{1}=s B$ for a local functional $B$ at ghost number -1 is of course a solution, but it is trivial in that it can be obtained from $W_{0}$ by means of a generalized non-linear field redefinition, where by "generalized" we mean one that may involve the ghosts and antifields as well. At the level of the original action such transformations translate into redefinitions of the field variables and/or gauge parameters. This leads to the conclusion that non-trivial first order (in the deformation parameter $g$ ) solutions of the master equation belong to the local cohomological group of $s$ at ghost number zero, denoted by $H^{0, D}(s \mid d)$. The same considerations apply to all higher order deformations. For instance $W_{2}$ now satisfies an inhomogeneous equation, but once a particular solution is found the homogeneous part will admit trivial terms arising from field redefinitions of $W_{2}$.

\section{Set-up}

We will classify all the consistent deformations of the free theory describing an arbitrary number of PM spin-2 fields: $h_{\mu \nu}^{a}, a=1, \ldots, n$. This theory is defined via the action

$$
S_{0}\left[h_{\mu \nu}^{a}\right]=\int d^{D} x \sqrt{-\bar{g}} k_{a b}\left(-\frac{1}{4} F^{a \lambda \mu \nu} F_{\lambda \mu \nu}^{b}+\frac{1}{2} F^{a \lambda} F_{\lambda}^{b}\right),
$$

where $\bar{g}_{\mu \nu}$ is the metric of the background that we choose to be (A)dS $\mathrm{d}_{D}$ space, $F_{\lambda \mu \nu}^{a}:=$ $2 \nabla_{[\lambda} h_{\mu] \nu}^{a}$ are the field strengths of the fields $h_{\mu \nu}^{a}, F^{a \lambda}:=\bar{g}_{\mu \nu} F^{\lambda \mu \nu}$ and $k_{a b}$ is a metric in the internal space of the PM spin-2 fields. Redefining the fields by means of the Gram-Schmidt process, one can take $\left(k_{a b}\right)=\operatorname{diag}(+\cdots+)$ for an unitary theory, which is only possible in $\mathrm{dS}_{D}$ space, and $\left(k_{a b}\right)=\operatorname{diag}(+\cdots+-\cdots-)$ for a non-unitary one. The field strengths and thus the action are invariant under the gauge transformations

$$
\delta_{\epsilon}^{(0)} h_{\mu \nu}^{a}=\nabla_{\mu} \nabla_{\nu} \epsilon^{a}-\frac{\sigma}{L^{2}} \bar{g}_{\mu \nu} \epsilon^{a},
$$

where $L$ is the $(\mathrm{A}) \mathrm{dS}_{D}$ radius related to the cosmological constant via $\Lambda=-\frac{(D-1)(D-2)}{2 \sigma L^{2}}$. The parameter $\sigma$ is defined to be +1 when the background is $\operatorname{AdS}_{D}$ and -1 when it is $\mathrm{dS}_{D}$. In this way the commutator of Lorentz-covariant derivatives on $(\mathrm{A}) \mathrm{dS}_{D}$ acting on a (co-) vector is given by

$$
\left[\nabla_{\mu}, \nabla_{\nu}\right] V_{\alpha}=-\frac{2 \sigma}{L^{2}} \bar{g}_{\alpha[\mu} V_{\nu]}
$$

In the BRST-BV formalism, we introduce the ghosts $C^{a}$ associated with the gauge parameters $\epsilon^{a}$ as well as the conjugate antifields $\left\{\Phi_{I}^{*}\right\}:=\left\{h_{a}^{* \mu \nu}, C_{a}^{*}\right\}$ canonically paired to 
the fields $\left\{\Phi^{I}\right\}:=\left\{h_{\mu \nu}^{a}, C^{a}\right\}$. The variance of the fields and antifields with respect to the color indices is by definition as displayed above up to raising or lowering indices thanks to the internal metric $k_{a b}$.

The BV functional of this theory is written as

$$
W_{0}=S_{0}+\int d^{D} x \sqrt{-\bar{g}}\left[h_{a}^{* \mu \nu}\left(\nabla_{\mu} \nabla_{\nu} C^{a}-\frac{\sigma}{L^{2}} \bar{g}_{\mu \nu} C^{a}\right)\right],
$$

and satisfies the classical master equation (2.3) as desired. We are then able to deduce the action of the BRST differential $s=\gamma+\delta$ on the fields and antifields:

$$
\begin{aligned}
s h_{\mu \nu}^{a} & =\gamma h_{\mu \nu}^{a}=\sqrt{-\bar{g}}\left(\nabla_{\mu} \nabla_{\nu} C^{a}-\frac{\sigma}{L^{2}} \bar{g}_{\mu \nu} C^{a}\right), \\
s h_{a}^{* \mu \nu} & =\delta h_{a}^{* \mu \nu}=\sqrt{-\bar{g}}\left(\nabla_{\lambda} F^{b \lambda(\mu \nu)}-\bar{g}^{\mu \nu} \nabla_{\lambda} F^{b \lambda}+\nabla^{(\mu} F^{\nu) b}\right) k_{a b}, \\
s C^{* a} & =\delta C^{* a}=\sqrt{-\bar{g}}\left(\nabla_{\mu} \nabla_{\nu} h_{a}^{* \mu \nu}-\frac{\sigma}{L^{2}} h_{a}^{*}\right),
\end{aligned}
$$

and 0 on the other ones.

The cohomology of the differential $\gamma$, which will be helpful in the following, is given by

$$
H(\gamma) \cong\left\{f\left(\left[F_{\lambda \mu \nu}^{a}\right], C^{a}, \nabla_{\mu} C^{a},\left[\Phi_{I}^{*}\right]\right)\right\} .
$$

\section{Cubic deformations}

The goal of this section is the classification of the first-order deformation of the BV functional $W_{1}$ which has to satisfy the master equation to first order (2.14). Expanding the first-order BV functional according to the antifield number

$$
W_{1}=\int d^{D} x \sqrt{-\bar{g}}\left(a_{0}+a_{1}+a_{2}\right),
$$

the master equation to first order is equivalent to the following descent equations ${ }^{3}$

$$
\begin{aligned}
\delta a_{1}+\gamma a_{0} & =t . d ., \\
\delta a_{2}+\gamma a_{1} & =t . d ., \\
\gamma a_{2} & =0 .
\end{aligned}
$$

\subsection{Deformations of the gauge algebra}

We will start the classification of the deformations by listing all the possible deformations of the gauge algebra $a_{2} \in H(\gamma)$, because $a_{2}$ has to be $\gamma$-closed in order to solve the equation (4.4) but not $\gamma$-exact in order not to be related to a trivial redefinition of the gauge parameters. Without any restriction on the number of derivatives, the complete list of candidates is given by

$$
\begin{aligned}
& a_{2}^{(1)}=C_{a}^{*} C^{b} C^{c} m_{b c}^{a}, \quad m_{b c}^{a}=m^{a}{ }_{[b c]}, \\
& a_{2}^{(2)}=C_{a}^{*} \nabla_{\mu} C^{b} \nabla^{\mu} C^{c} n_{b c}^{a}, \quad n_{b c}^{a}=n^{a}{ }_{[b c]},
\end{aligned}
$$

\footnotetext{
${ }^{3}$ Here $t . d .=\partial_{\mu} j^{\mu}$ for some vector $j^{\mu}$. Since one can always rewrite $j^{\mu}=\sqrt{-\bar{g}} \tilde{j}^{\mu}$ this implies $\partial_{\mu} j^{\mu}=$ $\sqrt{-\bar{g}} \nabla_{\mu} \tilde{j}^{\mu}$ and the $t . d$. represents up to a $\sqrt{-\bar{g}}$ factor a total derivative using a Lorentz-covariant derivative of the background. We will thus always write $t . d$. although this can mean $\partial_{\mu} j^{\mu}$ or $\nabla_{\mu} j^{\mu}$ depending on the context.
} 
and the total deformation of the algebra that solves the last equation of the descent (4.4) is a linear combination of all these candidates

$$
a_{2}=\alpha_{(1)} a_{2}^{(1)}+\alpha_{(2)} a_{2}^{(2)} .
$$

We now have to solve the second equation of the descent (4.3) with the $a_{2}$ just defined. The calculation of the two contributions to $\delta a_{2}$ gives

$$
\begin{aligned}
& \frac{1}{\sqrt{-\bar{g}}} \delta a_{2}^{(1)}=t . d .-\frac{1}{\sqrt{-\bar{g}}} \gamma\left[h_{a}^{* \mu \nu} m^{a}{ }_{b c} h_{\mu \nu}^{b} C^{c}\right]+m_{b c}^{a}\left(2 h_{a}^{* \mu \nu} \nabla_{\mu} C^{b} \nabla_{\nu} C^{c}+\frac{\sigma}{L^{2}} h_{a}^{*} C^{b} C^{c}\right), \\
& \frac{1}{\sqrt{-\bar{g}}} \delta a_{2}^{(2)}=t . d .-\frac{1}{\sqrt{-\bar{g}}} \gamma\left[2 n^{a}{ }_{b c} h_{a}^{* \mu \nu}\left(\nabla_{\mu} h_{\nu \rho}^{b} \nabla^{\rho} C^{c}+h_{\mu \rho}^{b} \nabla_{\nu} \nabla^{\rho} C^{c}+\frac{\sigma}{L^{2}} h_{\mu \nu}^{b} C^{c}\right)\right] \\
& +\frac{\sigma}{L^{2}} n_{b c}^{a}\left(2 h_{a}^{* \mu \nu} \nabla_{\mu} C^{b} \nabla_{\nu} C^{c}+\frac{\sigma}{L^{2}} h_{a}^{*} C^{b} C^{c}\right)-\frac{\sigma}{L^{2}} h_{a}^{*} n^{a}{ }_{b c} \nabla_{\mu} C^{b} \nabla^{\mu} C^{c} .
\end{aligned}
$$

The obstructions $2 h_{a}^{* \mu \nu} m^{a}{ }_{b c} \nabla_{\mu} C^{b} \nabla_{\nu} C^{c}+\frac{\sigma}{L^{2}} h_{a}^{*} m^{a}{ }_{b c} C^{b} C^{c} \in H(\gamma)$ and $2 h_{a}^{* \mu \nu} n^{a}{ }_{b c} \nabla_{\mu} C^{b} \nabla_{\nu} C^{c}$ $+\frac{\sigma}{L^{2}} h_{a}^{*} n^{a}{ }_{b c} C^{b} C^{c} \in H(\gamma)$ can be cancelled by relating $\alpha_{(2)}$ to $\alpha_{(1)}$ and $n^{a}{ }_{b c}$ to $m^{a}{ }_{b c}$. However, because of the obstruction $\frac{\sigma}{L^{2}} h_{a}^{*} n^{a}{ }_{b c} \nabla_{\mu} C^{b} \nabla^{\mu} C^{c} \in H(\gamma)$ arising in the calculation of $\delta a_{2}^{(2)}$, there exists no linear combination such that all the obstructions vanish. This implies that there is no solution to the inhomogenous (with $a_{2} \neq 0$ ) equation (4.3) and therefore we set $\alpha_{(1)}=0=\alpha_{(2)}$. In conclusion, the cubic deformations of "colored" PM fields are necessarily abelian.

\subsection{Abelian deformations of the gauge transformation}

We can still solve the homogeneous $\left(a_{2}=0\right)$ equation (4.3) by classifying all the possible abelian deformations of the gauge transformations $\bar{a}_{1} \in H(\gamma)$. In terms of coefficients $f_{(i) b c}^{a}, i=1, \ldots, 6$, there are six candidates containing up to two derivatives:

$$
\begin{array}{ll}
\bar{a}_{1}^{(1)}=h_{a}^{* \mu \nu} f_{(1) b c}^{a} \nabla_{\mu} F_{\nu}^{b} C^{c}, & \bar{a}_{1}^{(2)}=h_{a}^{* \mu \nu} f_{(2) b c}^{a} F_{\mu}^{b} \nabla_{\nu} C^{c}, \\
\bar{a}_{1}^{(3)}=h_{a}^{* \mu \nu} f_{(3) b c}^{a} \nabla^{\sigma} F_{\sigma \mu \nu}^{b} C^{c}, & \bar{a}_{1}^{(4)}=h_{a}^{* \mu \nu} f_{(4) b c}^{a} F_{\sigma \mu \nu}^{b} \nabla^{\sigma} C^{c}, \\
\bar{a}_{1}^{(5)}=h_{a}^{*} f_{(5) b c}^{a} \nabla_{\sigma} F^{b \sigma} C^{c}, & \bar{a}_{1}^{(6)}=h_{a}^{*} f_{(6) b c}^{a} F^{b \sigma} \nabla_{\sigma} C^{c} .
\end{array}
$$

An important aspect to remark is that not all combinations of these candidates are non-trivial because some combinations are $\delta$-exact. For example, the combination $\bar{a}_{1}^{(1)}+$ $\bar{a}_{1}^{(3)}-\bar{a}_{1}^{(5)}$ is $\delta$-exact when $f_{(3) b c}^{a}=f_{(5) b c}^{a}=f_{(1) b c}^{a}$ and $k_{a d} f_{(1) b c}^{a}=k_{a[d} f_{(1) b] c}^{a}$. Those particular combinations have to be removed from the possible non-trivial deformations.

\subsection{Cubic vertices}

We have to solve the first equation of the descent (4.2) with $a_{1}$ being the linear combination of all the non-trivial candidates written above. In the cases of, respectively, a single, two or three PM fields, there are 6, 48 or 162 candidates written in (4.10)-(4.12) (minus the $\delta$-exact combinations). The resolution of the equation (4.2) has been done systematically by listing all the possible vertices entering in $a_{0}$ containing no more than two derivatives and then searching for a set of solutions to the equation. ${ }^{4}$

\footnotetext{
${ }^{4}$ Note that allowing for more derivatives we can trivially construct Born-Infeld type vertices; they contain at least three derivatives and do not deform the gauge transformation laws of the free theory.
} 
We find that, provided $D=4$, the general solution is given by a vertex of the type "gauge field $\times$ conserved current":

$$
D=4: \quad a_{0}=h_{\mu \nu}^{a} J_{a}^{\mu \nu}, \quad a=1, \ldots, n,
$$

where

$$
J_{a}^{\mu \nu}:=\left(F_{\rho \sigma}^{b(\mu \mid \rho \sigma} F^{c \mid \nu)}-\frac{1}{4} \bar{g}^{\mu \nu} F^{b \rho \sigma \lambda} F_{\rho \sigma \lambda}^{c}-F^{b(\mu \mid} F^{c \mid \nu)}+F^{b(\mu|\sigma| \nu)} F_{\sigma}^{c}+\frac{1}{2} \bar{g}^{\mu \nu} F_{\lambda}^{b} F^{c \lambda}\right) f_{b c, a} .
$$

This vertex solves the descent equation with

$$
a_{1}=\bar{a}_{1}^{(4)}=h_{a}^{* \mu \nu} f_{b, c}^{a} F_{\sigma \mu \nu}^{b} \nabla^{\sigma} C^{c} .
$$

We write the coefficients $f_{a b, c}$ in this way because they have to satisfy

$$
f_{a b, c}=f_{(a b), c}
$$

in order to be related to a non-trivial solution of (4.2). The easiest way to see that is by noticing that the current $J_{a}^{\mu \nu}$ is on-shell ${ }^{5}$ equivalent to

$$
J_{a}^{\prime \mu \nu}=\left(F_{\rho \sigma \mid \rho \sigma}^{b(\mu \mid \nu)}{ }_{\rho \sigma}^{c-\frac{1}{4}} \bar{g}^{\mu \nu} F^{b \rho \sigma \lambda} F_{\rho \sigma \lambda}^{c}\right) f_{b c, a} \approx J_{a}^{\mu \nu},
$$

from which we see that the constants $f_{a b, c}$ are projected on $f_{(a b), c}$. The conclusions on the existence of vertices related to the current $J_{a}^{\prime \mu \nu}$ are unchanged if we use the current $J_{a}^{\mu \nu}$, but it is important to use the unprimed one in order to have a two-derivative gauge transformation. We remark that this primed current was already known in the case of a single PM field in the context of couplings to a massless spin-2 field [21]. The number of deformation parameters of this solution is thus given by the number of parameters encoded in $f_{a b, c}$, that is to say, $\frac{n^{2}(n+1)}{2}$.

Let us check that the vertex (4.13) is indeed consistent and how to reconstruct the gauge transformation (4.15). Since the current is manifestly gauge invariant because it is built out of the field strength, we have

$$
\frac{1}{\sqrt{-\bar{g}}} \gamma a_{0}=\left(\nabla_{\mu} \nabla_{\nu} C^{a}-\frac{\sigma}{L^{2}} \bar{g}_{\mu \nu} C^{a}\right) J_{a}^{\mu \nu}=t . d .+C^{a}\left(\nabla_{\mu} \nabla_{\nu} J_{a}^{\mu \nu}-\frac{\sigma}{L^{2}} \bar{g}_{\mu \nu} J_{a}^{\mu \nu}\right) .
$$

This implies through (4.2) that a necessary and sufficient ${ }^{6}$ condition to the consistency of the cubic vertex is that the current is conserved in the sense of the free PM gauge symmetry

$$
\nabla_{\mu} \nabla_{\nu} J_{a}^{\mu \nu}-\frac{\sigma}{L^{2}} J_{a} \approx 0 .
$$

Using the consequences of the equations of motion , mainly

$$
\begin{aligned}
F_{a}^{\nu} & =-\frac{\sigma L^{2}}{D-2} \nabla_{\mu} \frac{\delta S_{0}}{\delta h_{\mu \nu}^{a}} \approx 0 \\
\nabla_{\sigma} F_{a}^{\sigma \mu \nu} & =\frac{\delta S_{0}}{\delta h_{\mu \nu}^{a}}-\frac{\sigma L^{2}}{D-2} \bar{g}^{\mu \nu} \nabla_{\lambda} \nabla_{\sigma} \frac{\delta S_{0}}{\delta h_{\lambda \sigma}^{a}}+\frac{\sigma L^{2}}{D-2} \nabla^{\nu} \nabla_{\sigma} \frac{\delta S_{0}}{\delta h_{\mu \sigma}^{a}} \approx 0, \\
\nabla_{\sigma} F_{a}^{\mu \nu \sigma} & =\frac{2 \sigma L^{2}}{D-2} \nabla^{[\mu} \nabla_{\sigma} \frac{\delta S_{0}}{\delta h_{\nu] \sigma}^{a}} \approx 0,
\end{aligned}
$$

\footnotetext{
${ }^{5}$ We use the symbol $\approx$ for on-shell equalities, that is to say equalities on the surface of the equations of motion of the free theory.

${ }^{6}$ The condition is sufficient if one allows for higher-derivative terms in the gauge transformation.
} 
one finds that the current is on-shell divergenceless $\nabla_{\mu} J_{a}^{\mu \nu} \approx 0$ in arbitrary spacetime dimension as soon as the constants $f_{a b, c}$ are projected on $f_{(a b), c}$. Hence, since the current is divergenceless on-shell, it is required to be also traceless on-shell in order to solve (4.19). The trace of the current is given by

$$
J_{a}=\left(1-\frac{D}{4}\right) F_{\mu \rho \sigma}^{b} F^{c \mu \rho \sigma} f_{b c, a}+\left(\frac{D}{2}-2\right) F_{\mu}^{b} F^{c \mu} f_{b c, a}
$$

which turns out to be identically traceless only when $D=4$, and on-shell tracefull for $D \neq 4$.

In order to have access to $\bar{a}_{1}$ one needs to express all the on-shell terms arising in the calculation of the LHS of equation (4.19) using the consequences of the equations of motion (4.20)-(4.22) and $\frac{\delta S_{0}}{\delta h_{\mu \nu}^{a}}=\frac{1}{\sqrt{-\bar{g}}} \delta h_{a}^{* \mu \nu}$. One can show that

$$
\begin{aligned}
\nabla_{\mu} J_{a}^{\mu \nu} & =\frac{1}{\sqrt{-\bar{g}}} \delta h^{* b \rho \sigma} F_{\rho \sigma}^{c \nu} f_{b c, a}+\nabla_{\mu} A_{a}^{[\mu \nu]}, \\
A_{a}^{[\mu \nu]} & :=\frac{\sigma L^{2}}{2(D-2)} \nabla^{\alpha}\left(\frac{\delta S_{0}}{\delta h_{b}^{\alpha \rho}}\right) F^{c \nu \mu \rho} f_{b c, a} .
\end{aligned}
$$

It is remarkable that all the higher-derivative terms in $\nabla_{\mu} J_{a}^{\mu \nu}$ appear as $\nabla_{\mu} A_{a}^{[\mu \nu]}$ and this is due to the on-shell terms present in the current. Then one can reconstruct $\bar{a}_{1}$ via (4.2) to get

$$
\begin{aligned}
\frac{1}{\sqrt{-\bar{g}}} \gamma a_{0} & =\text { t.d. }-\nabla_{\nu} C^{a} \nabla_{\mu} J_{a}^{\mu \nu}=t . d .-\nabla_{\nu} C^{a}\left(\frac{1}{\sqrt{-\bar{g}}} \delta h^{* b \rho \sigma} F_{\rho \sigma}^{c \nu} f_{b c, a}+\nabla_{\mu} A_{a}^{[\mu \nu]}\right) \\
& =t . d .-\frac{1}{\sqrt{-\bar{g}}} \delta \underbrace{\left(h^{* a \mu \nu} F_{\sigma \mu \nu}^{b} \nabla^{\sigma} C^{c} f_{a b, c}\right)}_{=: a_{1}}+\underbrace{\nabla_{\mu} \nabla_{\nu} C^{a} A_{a}^{[\mu \nu]}}_{=0} .
\end{aligned}
$$

\subsection{Global symmetries of the free PM action}

Each infinitesimal consistent deformation of the BV action that modifies the gauge transformation can be viewed as the gauging of a global symmetry of the undeformed theory as soon as there exists a Killing parameter of the initial theory. Thus we will investigate if the deformation found only when the spacetime dimension is 4 is the gauging of a rigid symmetry that is only present in dimension 4 or in arbitrary dimension.

The master equation to first order in deformation at antifield number 0 (4.2) is equivalent to the fact that the deformed action is invariant under the deformed gauge transformation to first order in the deformation

$$
\delta_{0} S_{1}+\delta_{1} S_{0}=0
$$

Let us write $\delta_{0} \varphi^{i}=R^{(0) i}{ }_{\alpha} \varepsilon^{\alpha}, \delta_{1} \varphi^{i}=R^{(1) i}{ }_{\alpha} \varepsilon^{\alpha}$ the gauge variations of the fields at order 0 and 1 , and $\bar{\varepsilon}^{\alpha}$ the solutions of the Killing equations $\bar{\delta}_{0} \varphi^{i}=R^{(0) i}{ }_{\alpha} \bar{\varepsilon}^{\alpha}=0$. Evaluating (4.27) in the case where the gauge parameter is fixed to be a solution of the Killing equation directly gives $\bar{\delta}_{1} S_{0}=0$, implying that this is a global symmetry of the initial action.

In the case of the deformation of the section 4.3, one can extract the rigid transformation from (4.15) :

$$
\bar{\delta}_{1} h_{\mu \nu}^{a}=f_{b, c}^{a} F_{\sigma \mu \nu}^{b} \nabla^{\sigma} \bar{\varepsilon}^{c}
$$


where $\bar{\varepsilon}^{c}$ is a solution of the PM Killing equation

$$
\nabla_{\mu} \nabla_{\nu} \bar{\varepsilon}^{a}-\frac{\sigma}{L^{2}} \bar{g}_{\mu \nu} \bar{\varepsilon}^{a}=0 .
$$

Explicit solutions of this equation are constructed in [22] when the spacetime dimension is 4 but one can straigtforwardly extend the procedure to arbitrary dimension. Thus, because we know that the deformation build in section 4.3 is consistent in 4 dimensions, this global transformation is assured to be a symmetry of the action only in dimension 4 .

In order to see if this global symmetry is present in arbitrary dimension or not, we will look at the conserved charge associated with this global symmetry via Noether's theorem. According to it, a global symmetry is related to a current density $\mathcal{J}^{\mu}$ which is conserved in the sense

$$
\partial_{\mu} \mathcal{J}^{\mu} \approx 0 .
$$

Then one can construct a conserved charge as

$$
\mathcal{Q}=\int_{t=c s t} d^{D-1} x \mathcal{J}^{0},
$$

which is conserved because of (4.30), Stokes' theorem and the fact that the fields are supposed to vanish at spatial infinity.

In the context of the deformation calculated in section 4.3, we have a current $J_{a}^{\mu \nu}$ that is conserved not in the sense of Noether's theorem but in the sense (4.19) of the PM gauge symmetry. More precisely we showed that it satisfies

$$
\begin{aligned}
\nabla_{\mu} J_{a}^{\mu \nu} & \approx 0, \\
J_{a} & \equiv 0, \quad \text { if } D=4, \\
J_{a} & \neq 0, \quad \text { if } D \neq 4 .
\end{aligned}
$$

Using this, it is possible to build a true Noether current as

$$
\mathcal{J}_{a b}^{\mu}:=\sqrt{-\bar{g}} J_{a}^{\mu \nu} \nabla_{\nu} \bar{\varepsilon}_{b},
$$

where $\bar{\varepsilon}_{b}$ is still a solution of (4.29). Indeed we have

$$
\partial_{\mu} \mathcal{J}_{a b}^{\mu}=\sqrt{-\bar{g}} \nabla_{\mu}\left(J_{a}^{\mu \nu} \nabla_{\nu} \bar{\varepsilon}_{b}\right) \approx \sqrt{-\bar{g}}\left(J_{a}^{\mu \nu} \nabla_{\mu} \nabla_{\nu} \bar{\varepsilon}_{b}\right)=\frac{\sigma}{L^{2}} \sqrt{-\bar{g}} J_{a} \bar{\varepsilon}_{b},
$$

which is 0 if and only if the spacetime dimension is 4 . In conclusion one can build a conserved charge via (4.31) only in 4 dimensions and the corresponding global symmetry is given in (4.28).

\section{Quartic deformations}

The goal of this section is to solve the master equation to second order in deformation (2.15). Expanding the second-order BV functional according to the antifield number $W_{2}=$ $\int d^{D} x \sqrt{-\bar{g}}\left(b_{0}+b_{1}\right)$, the master equation to second order is equivalent to the following descent equations

$$
\begin{aligned}
\delta b_{1}+\gamma b_{0} & =-\sqrt{-\bar{g}}\left(a_{1}, a_{0}\right)+t . d . \\
\gamma b_{1} & =-\frac{1}{2} \sqrt{-\bar{g}}\left(a_{1}, a_{1}\right)+t . d .
\end{aligned}
$$




\subsection{Descent equation at antifield number 1}

Let us start with the calculation of $-\frac{1}{2}\left(a_{1}, a_{1}\right)$ in order to solve the equation (5.2). We have

$$
\begin{aligned}
\frac{1}{2}\left(a_{1}, a_{1}\right) & =t . d .-\frac{1}{\sqrt{-\bar{g}}} \gamma\left[2 h^{* a \mu[\nu} \nabla^{\sigma]} C^{b} F_{\rho(\mu \nu)}^{c} h_{\sigma}^{d \rho} f_{a e, b} f_{c, d}^{e}\right] \\
& +2 h^{* a \mu[\nu} \nabla^{\sigma]} C^{b} \nabla_{\sigma} F_{\rho(\mu \nu)}^{c} \nabla^{\rho} C^{d} f_{a e, b} f_{c, d}^{e}+\frac{3 \sigma}{2 L^{2}} h^{* a \mu \nu} \nabla^{\sigma} C^{b} C^{d} F_{\sigma \mu \nu}^{c} f_{a e, b} f_{c, d}^{e}
\end{aligned}
$$

The only way to kill the obstruction $h^{* a \mu \nu} \nabla^{\sigma} C^{b} C^{d} F_{\sigma \mu \nu}^{c} f_{a e, b} f_{c, d}^{e} \in H(\gamma)$ is by imposing the constraints

$$
f_{a e, b} f_{c, d}^{e}=0 .
$$

Let us suppose that we have a solution to the constraints. Then it implies that there is no second-order deformation of the gauge transformation $\left(b_{1}=0\right)$.

\subsection{Descent equation at antifield number 0}

The calculation of the antibracket $\left(a_{1}, a_{0}\right)$ gives

$$
-\left(a_{1}, a_{0}\right)=\left(F_{\sigma \mu \nu}^{b} \nabla^{\sigma} C^{c} f_{b, c}^{a}\right)\left(J_{a}^{\mu \nu}+\frac{\delta J_{d}^{\rho \lambda}}{\delta h_{\mu \nu}^{a}} h_{\rho \lambda}^{d}\right)=J_{a}^{\mu \nu} F_{\sigma \mu \nu}^{b} \nabla^{\sigma} C^{c} f_{b, c}^{a}
$$

because the second term vanishes when the constraints (5.4) hold. The current $J_{a}^{\mu \nu}$ can be expanded as (4.14) and then the identities (4.20)-(4.22) can still help to rewrite terms containing a trace $\left(F_{\mu}^{a}\right)$ or a divergence $\left(\nabla_{\mu} F_{a}^{\mu \nu \sigma}\right.$ or $\left.\nabla_{\sigma} F_{a}^{\mu \nu \sigma}\right)$ of the field strength as $\delta$ exact terms. Those $\delta$-exact terms actually come from a solution $\bar{b}_{1}$ of the homogeneous equation (5.2): $\gamma \bar{b}_{1}=0$. Using this trick one can write

$$
-\left(a_{1}, a_{0}\right)=\frac{1}{\sqrt{-\bar{g}}} \delta \bar{b}_{1}+F^{a \mu \rho \lambda} F_{\rho \lambda}^{b \nu} F_{\sigma \mu \nu}^{c} \nabla^{\sigma} C^{d} f_{a b,}{ }^{e} f_{e c, d}
$$

for some $\bar{b}_{1}$ whose explicit form is not needed, except for the fact that it is proportional to $f_{a b}{ }^{e} f_{e c, d}$ if one renames properly the color indices. The last term is generically a non-trivial element of the cohomology of $\gamma$ modulo $\mathrm{d}$ and modulo other $\delta$-exact terms. Indeed, one can remove the freedom of having a total derivative by taking a variational derivative with respect to the ghost $C^{d}$, which will produce some $\delta$-exact terms when the derivative will act as a divergence of the field strength but also other terms manifestly in the cohomology of $\gamma$. As a consequence they represent obstructions that are killed by imposing a second set of constraints

$$
f_{a b,}^{e} f_{e c, d}=0
$$

If one is able to solve the 2 sets of constraints (5.4)-(5.7), it implies that the theory closes at cubic order and is fully consistent with respect to the gauge structure. Indeed, in that case, $\bar{b}_{1}=0$ and the equation (5.1) is solved with $b_{0}=0$. 


\subsection{Solutions to the constraints}

This section is devoted to the resolution of the two sets of constraints

$$
\begin{aligned}
& f_{a m, b} f_{n c, d} k^{m n}=0, \\
& f_{a b, m} f_{n c, d} k^{m n}=0 .
\end{aligned}
$$

Unfortunately these have no solution when the internal metric $k_{a b}$ is Euclidean. This can be seen by analyzing the constraints for the indices $c=a=\bar{a}$ and $d=b=\bar{b}$ fixed and $k_{a b}=\delta_{a b}$ :

$$
f_{\bar{a} m, \bar{b}} f_{n \bar{a}, \bar{b}} \delta^{m n}=\sum_{e=1}^{n}\left(f_{\bar{a} e, \bar{b}}\right)^{2}=0 \quad \Longrightarrow \quad f_{\bar{a} e, \bar{b}}=0, \forall \bar{a}, e, \bar{b} .
$$

It is also direct that there is no solution for a single PM field and this is related to the fact that the known [23] self-interaction of a PM spin-2 field is obstructed to second order [4]. The fact that the internal metric $k_{a b}$ cannot be Euclidean implies that some fields must carry negative energy and the theory is therefore classically non-unitary.

Totally symmetric solutions. - An explicit solution of the quadratic constraints, Eqs. (5.8) and (5.9), is given by

$$
f_{a b, c}=(N-1)^{\left(n_{g}\right)_{a b c} / 2},
$$

for the choice of internal metric $k_{a b}=\operatorname{diag}(+1, \ldots,+1,-1)$, and where $\left(n_{g}\right)_{a b c} \in\{0,1,2,3\}$ denotes the number of times that the index " $N$ " (corresponding to the "ghostly" field in our convention) appears in $f_{a b, c}$; for instance $f_{N N, N}=(N-1)^{3 / 2}$.

Another simple particular solution that is valid for all even $N$ is $f_{a b, c}=1, \forall a, b, c \in$ $\{1, \ldots, N\}$, with metric $k_{a b}=\operatorname{diag}(+1, \ldots,+1,-1, \ldots,-1)$ that has the same number of " +1 " and " -1 " in its entries. Notice that both these solutions give a totally symmetric $f_{a b, c}$, in which case the two constraints (5.8) and (5.9) are in fact equivalent.

For $N=2$ fields these two solutions reduce to $f_{a b, c}=1, \forall a, b, c \in\{1,2\}$, with metric $k_{a b}=\operatorname{diag}(+1,-1)$. In this case we can moreover show that this solution is unique modulo rescalings of the fields and the gauge parameters. We remark that for $N \geq 3$ the constants (5.11) lead to cubic vertices that couple three distinct fields, so that it is not a trivial extension of the $N=2$ solution.

Mixed symmetric solutions.-For $N=2$ the unique solution to the constraints was totally symmetric under the exchange of the three indices. However, for $N \geq 3$, there also exist solutions for mixed-symmetric constants $f_{a b, c}$. For example, when $N=3$ and the metric is $k_{a b}=\operatorname{diag}(+1,+1,-1)$, one such solution is given by

$$
\begin{aligned}
& f_{11,1}=f_{22,2}=1, \\
& f_{11,2}=-f_{12,1}=f_{22,1}=-f_{12,2}=1, \\
& f_{13,1}=f_{13,2}=-f_{23,1}=-f_{23,2}=\sqrt{2}, \\
& f_{33,1}=f_{33,2}=2, \\
& f_{a b, 3}=0 \quad \forall a, b \in\{1,2,3\} .
\end{aligned}
$$




\section{References}

[1] K. Hinterbichler, "Theoretical Aspects of Massive Gravity," Rev. Mod. Phys. 84 (2012) 671-710, arXiv:1105.3735 [hep-th].

[2] C. de Rham, "Massive Gravity," Living Rev. Rel. 17 (2014) 7, arXiv:1401.4173 [hep-th].

[3] C. de Rham, K. Hinterbichler, R. A. Rosen, and A. J. Tolley, "Evidence for and obstructions to nonlinear partially massless gravity," Phys. Rev. D88 (2013) no. 2, 024003, arXiv:1302.0025 [hep-th].

[4] E. Joung, W. Li, and M. Taronna, "No-Go Theorems for Unitary and Interacting Partially Massless Spin-Two Fields," Phys. Rev. Lett. 113 (2014) 091101, arXiv:1406.2335 [hep-th].

[5] S. Garcia-Saenz and R. A. Rosen, "A non-linear extension of the spin-2 partially massless symmetry," JHEP 05 (2015) 042, arXiv:1410.8734 [hep-th].

[6] G. Barnich and M. Henneaux, "Consistent couplings between fields with a gauge freedom and deformations of the master equation," Phys. Lett. B311 (1993) 123-129, arXiv:hep-th/9304057.

[7] I. Batalin and G. Vilkovisky, "Gauge Algebra and Quantization," Phys.Lett. B102 (1981) 27-31.

[8] I. Batalin and G. Vilkovisky, "Quantization of Gauge Theories with Linearly Dependent Generators," Phys.Rev. D28 (1983) 2567-2582.

[9] F. A. Berends, G. J. H. Burgers, and H. van Dam, "On the theoretical problems in constructing interactions involving higher spin massless particles ," Nucl. Phys. B260 (1985) 295.

[10] N. Boulanger, C. Deffayet, S. Garcia-Saenz, and L. Traina, "Theory for multiple partially massless spin-2 fields," Phys. Rev. D100 (2019) no. 10, 101701, arXiv:1906.03868 [hep-th].

[11] N. Boulanger, A. Campoleoni, and I. Cortese, "Dual actions for massless, partially-massless and massive gravitons in (A)dS," Phys. Lett. B782 (2018) 285-290, arXiv:1804.05588 [hep-th].

[12] M. Henneaux, "Consistent interactions between gauge fields: The cohomological approach," Contemp. Math. 219 (1998) 93, arXiv:hep-th/9712226.

[13] N. Boulanger, T. Damour, L. Gualtieri, and M. Henneaux, "Inconsistency of interacting, multigraviton theories," Nucl. Phys. B597 (2001) 127-171, arXiv:hep-th/0007220. 
[14] C. Becchi, A. Rouet, and R. Stora, "Renormalization of Gauge Theories," Annals Phys. 98 (1976) 287-321.

[15] I. Tyutin, "Gauge Invariance in Field Theory and Statistical Physics in Operator Formalism," arXiv:0812.0580 [hep-th].

[16] N. Boulanger and M. Esole, "A Note on the uniqueness of $\mathrm{D}=4, \mathrm{~N}=1$ supergravity," Class. Quant. Grav. 19 (2002) 2107-2124, arXiv:gr-qc/0110072 [gr-qc].

[17] N. Boulanger, B. Julia, and L. Traina, "Uniqueness of $\mathcal{N}=2$ and 3 pure supergravities in 4D," JHEP 04 (2018) 097, arXiv:1802.02966 [hep-th].

[18] J. Gomis, J. Paris, and S. Samuel, "Antibracket, antifields and gauge theory quantization," Phys.Rept. 259 (1995) 1-145, arXiv:hep-th/9412228 [hep-th].

[19] G. Barnich, N. Boulanger, M. Henneaux, B. Julia, V. Lekeu, and A. Ranjbar, "Deformations of vector-scalar models," JHEP 02 (2018) 064, arXiv:1712.08126 [hep-th].

[20] G. Barnich and N. Boulanger, "A note on local BRST cohomology of Yang-Mills type theories with free abelian factors," J. Math. Phys. 59 (2018) 052302, arXiv:1802.03619 [hep-th].

[21] E. Joung, K. Mkrtchyan, and G. Poghosyan, "Looking for partially-massless gravity," JHEP 07 (2019) 116, arXiv:1904.05915 [hep-th].

[22] K. Hinterbichler and R. A. Rosen, "Partially massless monopoles and charges," Phys.Rev.D 92 (2015) no. 10, 105019, arXiv:1507.00355 [hep-th].

[23] Yu. M. Zinoviev, "On massive spin 2 interactions," Nucl. Phys. B770 (2007) 83-106, arXiv:hep-th/0609170 [hep-th]. 